\title{
Adaptação e evidências de validade do Recovery-Stress Questionnaire for Athletes (RESTQ-Sport) para dançarinos adolescentes (RESTQ-Dance)
}

Andressa Melina Becker da Silva. Universidade de Sorocaba.

Sônia Regina Fiorim Enumo. Pontifícia Universidade Católica de Campinas.

Murilo Fernandes de Araújo. Pontifícia Universidade Católica de Campinas.

Lucas de Francisco Carvalho. Universidade de São Francisco.

Isabella Goulart Bittencourt. Universidade Federal Santa Catarina.

Renan de Morais Afonso. Pontifícia Universidade Católica de Campinas.

Tatiane Stephan Rocchetti Luz. Pontifícia Universidade Católica de Campinas.

\section{Resumo}

O estresse ocorre na transação indivíduo-ambiente quando eventos são avaliados como excedendo a capacidade de enfrentá-los e ameaçam o bem-estar. Para sua avaliação no contexto da dança, há poucos instrumentos. Este estudo adaptou para dançarinos adolescentes o questionário Recovery-Stress Questionnaire for Athletes (RESTQ-Sport), verificando-se suas evidências de validade. Foram avaliados 264 dançarinos brasileiros, de ambos os sexos (93,18\% mulheres), com idade média de 14,69 anos, durante festivais de dança de nível nacional e internacional. Verificaram-se as evidências de validade de conteúdo, com auxílio de juízes, e evidências de validade de consistência interna (análise fatorial e alfa de Cronbach). O RESTQ-Dance ficou com 63 itens, organizados em três fatores: Fator 1 - Estresse Geral (26 itens, $\alpha$ = 0,92); Fator 2 - Recuperação (27 itens, $a=0,91$ ); Fator 3 - Estresse Específico (10 itens, $a=0,79$ ); sendo excluídos 14 itens nesse processo. $\mathrm{O}$ instrumento apresenta propriedades psicométricas adequadas para uso com dançarinos.

Palavras-chave: estresse; recuperação; dança; testes psicológicos.

\begin{abstract}
Aaptation and vality evidences of Recovery-Stress Questionnaire for Athletes (RESTQ-Sport) for dancers (RESTQ-Dance). Stress occurs in the individual-environment transaction, when events are assessed as exceeding the ability to face them and threaten the well-being. For evaluation in the context of dance, there are few instruments. This study adapted for adolescent dancers the Recovery-Stress Questionnaire for Athletes (RESTQ-Sport), verifying their validity evidence. Have been evaluated 264 Brazilian dancers, male and female (93.18\% women) with a mean age of 14.69 years, during national and international levels of dance festivals. There has been validity evidence of content, with the help of judges, and validity evidence of internal consistency (factor analysis and Cronbach's alpha). The RESTQ-Dance was left with 63 items organized into three factors: Factor 1 - Stress General (26 items, $a=0.92$ ); Factor 2 - Recovery (27 items, $a=0.91$ ); Factor 3 - Specific Stress (10 items, $a=0.79$ ); being excluded 14 items in this process. The instrument has adequate psychometric properties for use with dancers.
\end{abstract}

Keywords: stress; recover; dance; psychological tests.

\section{Resumen}

Adaptación y evidencias de validez el Recovery-Stress Questionnaire for Athletes (RESTQ-Sport) para bailarines (RESTQ-Dance). El estrés se produce en la transacción con el medio individual, cuando los eventos son evaluados como superior a la capacidad de enfrentarse a ellos y poner en peligro el bienestar. Para la evaluación en el contexto de la danza, hay pocos instrumentos. En este estudio se adaptó para adolescentes bailarines el cuestionario Recovery-Stress Questionnaire for Athletes (RESTQ-Sport), verificando su evidencia de validez. Se evaluaron 264 bailarines brasileños, hombres y mujeres (93,18\% mujeres) con una edad media de 14,69 años para los festivales de nivel nacionales y internacionales de danza. Se verificó evidencia de la validez de contenido, con la ayuda de los jueces, y la evidencia de validez de consistencia interna (análisis factorial y alfa de Cronbach). EI RESTQ-Dance se quedó con 63 artículos organizados en tres factores: Factor 1 - Estrés generales (26 ítems, $\alpha=0$,92); Factor 2 - Recuperación (27 ítems, $a=0$,91); Factor 3 - Estrés específico (10 ítems, $a=0,79$ ); siendo excluidos 14 ítems en este proceso. El instrumento tiene propiedades psicométricas adecuadas para su uso con los bailarines.

Palabras clave: estrés; recuperación; baile; pruebas psicológicas. 
O dançarino profissional de alto rendimento frequentemente se encontra sob excessiva pressão física e psicológica, por atuar em meio a um ambiente extremamente competitivo. É um padrão que já acompanha os atletas, cuja carga de treinamento média aumentou $20 \%$ a partir da década de 90 , com grande parte dos atletas de alto nível demonstrando sintomas de overtraining (excesso de treinamento), overreaching (acúmulo de treinamento) e burnout (respostas psicofísiológicas ao estresse ocupacional severo) (Meehan, Bull, Wood, \& James, 2004). Estas são respostas de estresse exacerbadas, as quais têm sido tradicionalmente compreendidas pelo modelo cognitivista ou transacional do estresse, proposto por Lazarus e Folkman (1984). O estresse é entendido como uma relação indivíduo-ambiente avaliada como excedendo seus recursos de enfrentamento. Este modelo inclui, assim, as estratégias de enfrentamento do estresse (coping), considerando as transações indivíduoambiente de uma maneira multidirecional. Por ser uma reação particular, as pessoas interpretam uma mesma situação de maneira diferenciada, de forma que o mesmo evento pode ser muito estressante para algumas e não para outras. Pode, então, afetar o bem-estar físico e psicológico, necessitando de respostas que ajudem a restaurar o equilíbrio. Contudo, ao se estudar o estresse, o importante não é somente avaliar se ele é bom ou ruim, mas a intensidade e o momento do desenvolvimento em que ele é prejudicial ou útil, considerando-se este processo, a princípio, como adaptativo. Seu desfecho na saúde física e mental, no médio e longo prazo, é que deve servir como parâmetro para avaliação de sua adequação em termos adaptativos.

Atualmente, considera-se haver três tipos de estresse, segundo Shonkoff et al. (2012): a) o estresse "positivo", que é moderado e curto, causa pequenos aumentos no batimento cardíaco e nos níveis de hormônios relacionados ao estresse; b) o estresse "tolerável", severo o bastante para prejudicar a arquitetura do cérebro infantil/adolescente caso não seja controlado, sendo, porém, amenizado por relacionamentos de apoio que facilitem o enfrentamento e diminua seus efeitos deletérios. Na maioria dos casos, o estresse tolerável ocorre apenas durante um curto período de tempo, possibilitando o cérebro a se recuperar de efeitos danosos; e c) o estresse "tóxico", que ocorre quando é severo e prolongado e sem suporte do adulto. Este último tipo de estresse causa danos na arquitetura cerebral e leva a problemas permanentes de aprendizagem, comportamento e saúde mental e física.
No contexto esportivo e de atividade física, diversos estressores podem estar agregados, seja aos treinamentos, seja em competições. De acordo com Urhausen e Kindermann (2002), na situação de treinamento, o aumento gradativo na rotina de treino é algo comum, como forma de estimular adaptação do indivíduo e obter melhores resultados. Contudo, caso esse aumento aconteça de maneira abrupta e o sujeito não consiga se adaptar a tempo, pode ocorrer uma sobrecarga, que gera fadiga e queda no rendimento. Para os mesmos autores, sem uma adequada recuperação dos treinamentos, os quais acontecem geralmente em alta intensidade e frequência, o atleta pode desenvolver sintomas de overtraining (excesso de treinamento), além de vivenciar o que se denomina de overreaching.

Overtraining refere-se a um desequilíbrio entre estresse e recuperação e pode ser caracterizado como uma síndrome, cujos sinais abrangem queda no desempenho, fadiga crônica, infecções respiratórias e alterações no humor (Alves, Costa, \& Samulski, 2006). Além disso, lesão, fraqueza muscular, problemas nutricionais, mudanças hormonais e depressão podem ser sintomas da síndrome do excesso de treinamento (G. S. Cunha, Ribeiro, \& Oliveira, 2006). Já o overreaching é definido pelo acúmulo de treinamento, como a busca de se induzir um desempenho máximo, mas que acaba por gerar um decréscimo de rendimento. Ambos os fenômenos causam a diminuição do desempenho e têm sintomas semelhantes. A principal diferença entre overtraining e overreaching está relacionada ao tempo de recuperação. No overreaching, são necessárias de duas a três semanas para o reestabelecimento do equilíbrio do atleta; já no excesso de treinamento (overtraining), esse processo pode acontecer em meses ou anos (Uusitalo, 2001). Associado aos fenômenos overtraining e overreaching, está o burnout, que se caracteriza por síndrome psicológica decorrente da forte tensão emocional crônica associada ao estresse ocupacional severo, apresentando uma resposta psicofisiológica mais completa de afastamento do ambiente competitivo e do treinamento excessivo (Verardi, Miyazaki, Nagamine, Lobo, \& Domingos, 2012).

No ambiente altamente competitivo da dança, que é estressante por si só (Rohleder, Beulen, Chen, Wolf, \& Kirschbaum, 2007; Silva, Tkac, \& Enumo, 2013), a situação se agrava quando se trata de adolescentes (Silva, 2016). Entre 10 e 18 anos de idade, ocorrem diversas transformações amplas e rápidas de contexto biológico, psicológico e social, devido à maturação sexual e busca de independência emocional e financeira da 
família (Breinbauer \& Maddaleno, 2008). Segundo Tricoli (2014), fatores estressantes externos contribuem para o agravamento da situação, como a relação com a família e o contexto escolar, e fatores estressantes internos, como necessidade de aceitação e preocupação por mudanças físicas.

Para atletas adolescentes, cinco principais estressores estão presentes: fatores intrínsecos do esporte, regras de organização esportiva, relacionamento esportivo e demandas interpessoais, carreira atlética e desenvolvimento de desempenho, e estrutura organizacional e clima esportivo (Fletcher, Hanton, Mellalieu, \& Neil, 2012). Muitas vezes, esses fatores estressantes se encontram intimamente ligados e são agravados pela presença de overtraining e burnout. Esses quadros foram encontrados na pesquisa de Barros e De Rose Jr. (2006), com 216 nadadores de 7 a 12 anos. Apesar de apresentar diferenças em relação aos treinamentos e competições, a dança se assemelha aos esportes (Guarino, 2015), indicando a necessidade de analisar os estressores e os níveis de estresse de dançarinos.

Levando em consideração o contexto da dança, na visão de Verderi (1998), a dança se caracteriza como a arte do movimento, pela qual o homem pode demonstrar papéis sociais e desempenhar relações dentro da sociedade. E, apesar da dança muitas vezes ser recomendada como uma maneira de se diminuir o estresse, Sousa, Mariani e Samulski (2004) relembram:

[...] quando se trata da apresentação de um espetáculo de dança, principalmente a pré-estréia, deve-se levar em consideração a influência de determinados fatores incluindo, dentre outros, o medo de errar, o esquecimento da seqüência coreográfica, a presença da platéia e de críticos da área. Desta maneira, os bailarinos profissionais poderiam estar sujeitos a pressões psicológicas, que os levam ao estresse e à ansiedade. (p. 5)

Como se vê, os dançarinos muitas vezes passam por situações extremamente estressantes, física e psicologicamente, visando se tornarem profissionais na área da dança, atingindo e mantendo um alto nível técnico, artístico e plástico. Nesse processo, podem ocorrer sintomas de overtraining, overreaching e burnout em grande parte da população praticante (Constantino, Prado, \& Lofrano-Prado, 2010; Koutedakis et al., 1999). Tendo em vista essa alta incidência, é importante fazer uma correta avaliação do nível de estresse dos dançarinos e de sua capacidade de recuperação, para que só então uma intervenção coerente seja realizada, visando ao equilíbrio entre estresse e recuperação, bem como o aprimoramento de estratégias de enfrentamento adaptativas ao estresse.

Contudo, há poucos estudos sobre avaliação psicológica na área da Psicologia do Esporte e da Atividade Física voltados para a dança. Isso gera problemas metodológicos e psicométricos, pois muitos instrumentos clínicos são usados nesta área sem adaptações (Silva \& Enumo, 2016). Rubio (2011) alerta para o risco do uso generalizado de instrumentos da área clínica para a área dos esportes, tendo em vista que estes não contemplam especificidades esportivas ou mesmo de características psicológicas importantes ao contexto. Há, portanto, pouca discussão sobre essa temática e falta de capacitação profissional para a construção de novos instrumentos específicos ou mesmo validação de outros. Autores da área (Peixoto \& Nakano, 2014; Silva, Guimarães, Foch, \& Enumo, 2014) também alertam para a inexistência de testes psicológicos aprovados para uso profissional, validados para atletas, fazendo com que os profissionais optem por utilização de testes sem requisitos de evidências de validade e confiabilidade.

Um dos instrumentos usados na área é o RESTQ-Sport (Recovery-Stress Questionnaire for Athletes) (Kellmann \& Kallus, 1993), que mede estresse e recuperação em atletas. É utilizado pelos Comitês Olímpicos Alemão e Americano como instrumento oficial de monitoramento de treinamento, além de ser utilizado em vários outros países de língua alemã e inglesa (L. O. Costa \& Samulski, 2005). No Brasil, foi adaptado e validado por L. O. Costa e Samulski (2005), com uma população de 134 atletas das modalidades de judô, ginástica artística (antiga ginástica olímpica) e natação. Tanto na validação brasileira, como no instrumento original, três de suas 19 escalas atingiram níveis satisfatórios de consistência interna - Sucesso, Conflito/Pressão e Aceitação Pessoal -, principalmente devido a diferentes vivências de atletas em modalidades individuais e coletivas. Já as outras escalas atingiram valores satisfatórios, alinhadas aos estudos internacionais.

Por ser um bom indicador de overtraining, o RESTQ-Sport tem sido utilizado em pesquisas nacionais (L. O. Costa \& Samulski, 2005; R. A. Cunha, Morales, \& Samulski, 2008; Freitas, Souza, Oliveira, Pereira, \& Nakamura, 2014; Machado, Matos, \& Samulski, 2011; Matos, Samulski, Lima, \& Prado, 2014; Nogueira et al., 2015) e internacionais (Brink et al., 2010; GonzálezBoto, Salgueiro, Tuero, \& Márquez, 2009; Kellmann, 
Altenburg, Lormes, \& Steinacker, 2001; Kellmann \& Günther, 2000; Jürimäe, Mäestu, \& Jürimäe, 2004), com amostras de atletas do futsal, basquete, rugbi, atletismo, ciclismo, escalada, futebol, grupos de corrida, nadadores, remadores, entre outros. Todos os estudos trazem discussões pertinentes à modalidade praticada, comparando os resultados obtidos com o instrumento validado originalmente. Portanto, as diferenças entre os tipos de modalidade de atividade física ou esportiva praticada - coletivas e individuais; de campo, quadra, rua; abertas e fechadas, por exemplo - devem ser consideradas, pois exigem habilidades físicas e psicológicas distintas. Estas devem ser levadas em consideração antes da aplicação de qualquer instrumento de avaliação psicológica, bem como na análise de seus resultados (McCann, Jowdy, \& Van Raalte, 2011). Percebe-se aqui a importância da validação de instrumentos específicos ou adaptados para as diferentes modalidades de atividade física/esportiva e para as diferentes situações no esporte (treinamento ou competição).

Apesar dessas aplicações do instrumento no país, sua validação foi feita somente para atletas de três modalidades (Judô, ginástica artística e natação), confirmando o quadro de que boa parte dos estudos nacionais não buscou realizar uma adaptação e validação para populações de diferentes modalidades esportivas. Com esse enfoque, o presente estudo pretendeu adaptar e verificar as evidências de validade de um instrumento voltado à avaliação do estresse e da recuperação de atletas - o Recovery-Stress Questionnaire for Athletes [RESTQ-Sport] (Kellmann \& Kallus, 2001, adaptado por L. O. Costa \& Samulski, 2005) - para bailarinos adolescentes.

\section{Método}

\section{Participantes}

O presente estudo foi composto por 264 dançarinos brasileiros participantes voluntários, de ambos os sexos (246 mulheres e 18 homens), com idade entre 10 e 19 anos (média =14,69; $\pm 1,85$ ), os quais dançavam, em média, há 8,05 anos $( \pm 3,96)$. O nível socioeconômico médio dos participantes foi classificado na classe B2 (média salarial familiar de $\mathrm{R} \$ 2.654,00$ reais) pelo Critério de Classificação Econômica Brasil (Associação Brasileira de Empresas de Pesquisa [ABEP], 2013). Eles compuseram uma amostra não probabilística, de conveniência, sendo selecionados em festivais de dança de nível nacional e internacional, no Estado de São Paulo e de Santa Catarina, conforme disponibilidade para participar do estudo e assinatura do termo de consentimento para participação em pesquisa. Foram critérios de inclusão: dançarinos com idade entre 10 e 19 anos, alfabetizados, e que tivesse a autorização dos pais ou responsáveis para participação na pesquisa. Como critérios de exclusão, consideraramse os dançarinos que competiam há menos de um ano, ou que não responderam alguns itens do instrumento. Inicialmente, foram incluídos 410 dançarinos, mas após os critérios de exclusão, restaram 264 dançarinos para as análises estatísticas (que haviam preenchido todos os itens do instrumento).

\section{Instrumentos}

O Recovery-Stress Questionnaire for Athletes [RESTQ-Sport] (Kellmann \& Kallus, 2001, adaptado por L. O. Costa \& Samulski, 2005) foi usado no presente estudo em bailarinos. Mede o estresse e a recuperação em atletas, com 77 questões, em uma escala de seis pontos (1 - Pouquíssimas Vezes a 6 - Sempre). É solicitado que o indivíduo leve em consideração os três últimos dias em relação a comportamentos, sentimentos e emoções vivenciados. $O$ instrumento original contém 19 fatores: Estresse Geral, Estresse Emocional, Estresse Social, Fadiga, Perda de Energia, Queixas Físicas, Sucesso, Recuperação Social, Recuperação Física, Bem-estar Geral, Qualidade de sono, Distúrbios nos intervalos, Exaustão Emocional, Lesões, Estar em Forma, Aceitação Pessoal, Autoeficácia e Autorregulação. São também computados valores totais: Escala de Estresse Geral, Escala de Estresse Específico, Escala de Estresse Global, Escala de Recuperação Geral, Escala Áreas de Recuperação, Escala Recuperação Global. Os valores de confiabilidade, mensurados pelo Alfa de Cronbach, variaram de 0,58 a 0,85 , sendo que 16 das 19 subscalas apresentaram fidedignidade significativa, ou seja, acima de 0,70 (L. O. Costa \& Samulski, 2005). Pela variação da escala entre 0 a 6, valores acima de 4 são considerados "altos", abaixo de 2 são considerados "baixos" e, entre 2,01 e 3,99, são "moderados", seja para estresse específico, estresse geral ou recuperação.

Foi também utilizado o Critério de Classificação Econômica Brasil (ABEP, 2013), um questionário usado em pesquisas para avaliação da classe socioeconômica, hierarquizada em ordem decrescente: A, B1, B2, C1, C2, $D, E$, em termos de poder aquisitivo (Classe A: renda média familiar bruta mensal $=R \$ 9.263,00$; classe $E: R \$$ 776,00). 


\section{Procedimento}

Os participantes foram selecionados durante festivais de dança de caráter nacional e internacional no Sul e Sudeste do Brasil, durante os meses de abril a setembro de 2014. A aplicação dos instrumentos ocorreu antes dos ensaios de marcação de palco (último treino antes da competição) por uma equipe de cinco aplicadores treinados. Para isso, foram utilizadas pranchetas como apoio, para que as respostas fossem dadas no ambiente de competição, tendo em vista que nenhuma sala foi disponibilizada para tal. A participação foi voluntária, tendo sido informados tanto o participante como o professor e/ou coreógrafo da companhia de dança sobre os objetivos do estudo, características do questionário, fatores de risco e a sigilosidade dos dados, pedindo para que ambos assinassem o Termo de Consentimento Livre e Esclarecido para participação em pesquisa, aprovada pelo Comitê de Ética da universidade (no 770.131). Os participantes foram instruídos a responder todos os itens, observando que, na folha de resposta, existiam perguntas em frente e verso; foi dito também que não havia respostas certas ou erradas, e que eles deveriam responder ao que mais se aproximasse da realidade deles, pensando nos últimos três dias, conforme instruções do próprio instrumento.

\section{Processamento e Análise de Dados}

Os autores Primi, Muniz e Nunes (2009) fizeram uma tradução da terminologia proposta pela American Educational Research Association, American Psychological Association e National Council for Measurement in Education [AERA/APA/NCME] (2014), denominando-a de "evidências de validade". Com base nas novas diretrizes psicométricas, foram utilizados os critérios de "evidências de validade", verificando o conteúdo frente aos juízes e a consistência interna frente a análises fatoriais. Inicialmente foi feita uma revisão em termos de linguagem do RESTQSport levando em consideração as características dos bailarinos e da faixa etária (adolescência) e de acordo com a experiência prática de 22 anos da primeira autora, modificando-se alguns termos, trocando-se os termos competição e treinamentos por festival/espetáculo e ensaios; equipe por grupo de dança.

Após esta adequação, seguiram-se alguns procedimentos empíricos, conforme proposição de Pasquali (2004), com a análise dos itens do instrumento realizada por cinco juízes (dois professores de português, um psicólogo do esporte, dois educadores físicos com especialidade em dança), de maneira a observar a análise semântica (compreensão dos itens) e pertinências prática e teórica do questionário. Utilizou-se um protocolo adaptado de Balbinotti, Benetti e Terra (2007, p. 11), em que cada juiz avalia cada item do instrumento perante clareza de linguagem, pertinência teórica e pertinência prática; além de poder sugerir alterações. Para se verificar a clareza de linguagem, a seguinte pergunta será feita aos juízes: "Você acha que estes itens são claros o suficiente e, portanto, serão entendidos pelos bailarinos? Em que extensão?". Para avaliar a pertinência prática, perguntou-se: "Você acha que estes itens são pertinentes para os bailarinos? Em que extensão?". No campo das observações do protocolo, os juízes puderam apontar falhas, sugerindo correções ou exclusão de determinados itens. Como opções de resposta, os juízes utilizaram uma escala de cinco pontos, sendo 1 = pouquíssima, 2 = pouca, 3 = média, 4 = muita, 5 = muitíssima .

Após a devolução das folhas de respostas dos juízes, realizou-se o cálculo do Coeficiente de Validade do Conteúdo (CVC) para clareza da linguagem e pertinência prática, conforme proposto por Hernández-Nieto (2002), descrito a seguir:

1. Calcular a média das notas de cada item (Mx) com base nas notas dos juízes:

$M x$ = soma das notas dos juízes/número de juízes que avaliaram o item

2. Com base na média obtida (Mx), calcular o CVC para cada item (CVCi):

$\mathrm{CVC}_{\mathrm{i}}=$ média das notas de cada item/valor máximo que o item poderia receber

3. Calcular o erro $\left(\mathrm{P}_{\mathrm{ei}}\right)$, para descontar possíveis vieses dos juízes avaliadores, para cada item: $\mathrm{P}_{\mathrm{ei}}=(1 /$ número de juízes que avaliaram o item) número de juízes que avaliaram o item

4. Aplicar a fórmula para o CVC final de cada item (CVCc):

$\mathrm{CVC}_{\mathrm{c}}=\mathrm{CVC}_{\mathrm{i}}-\mathrm{Pe}_{\mathrm{i}}$

5. Aplicar a fórmula para o cálculo do CVC total do questionário (CVCt), para cada uma das características (clareza de linguagem e pertinência prática):

$C V C_{t}=M c v c_{i}-M_{p e}$ onde: $M_{i c v c_{i}}=$ média dos coeficientes de validade de conteúdo dos itens do questionário; $\mathrm{Mpe}_{\mathrm{i}}=$ média dos erros dos itens do questionário.

Concluída a coleta de dados, estes foram tabulados e procedeu-se às análises estatísticas. Em 
relação à avaliação da dimensão teórica do instrumento, calculou-se o índice de concordância intra-avaliadores por meio do índice Kappa, realizando-se as correções necessárias.

O número de fatores a serem mantidos na análise fatorial exploratória foi verificado com base na análise paralela (Hayton, Allen, \& Scarpello, 2004; Watkins, 2006). Para veiculação da análise, foi utilizado o software R, versão 2.15.3, por este possibilitar o uso da análise paralela para variáveis policóricas, como é caso do presente estudo. Foi gerado um banco de dados para o software MPlus versão 6.12, para a análise fatorial exploratória, com índices de ajuste ao modelo com variáveis policóricas, além da obtenção de índices confirmatórios, que indicam a adequação da estrutura encontrada com base na presente amostra. Ressalta-se, ainda, que o ajustamento nos índices confirmatórios também sugere a adequação da amostra para a realização da análise.

\section{Resultados}

Os resultados serão apresentados segundo as evidências de validade de conteúdo e de estrutura interna para o RESTQ-Dance.

\section{Evidências de validade baseadas no conteúdo do RESTQ-Dance}

O RESTQ-Sport foi aplicado na versão validada para o português brasileiro por L. O. Costa e Samulski (2005) adaptada para o contexto de bailarinos adolescentes, conforme descrito no tópico anterior. Foram feitas alterações na redação dos itens adequando-a ao cotidiano do grupo de dança, tanto com a nomeação para situações comuns (termos, como "ensaio", "festival", "espetáculo", "modalidade de dança") e à faixa etária da adolescência.

A análise dos juízes apresentou uma concordância medida pelo índice Kappa de 0,82\% de pertinência teórica; $0,87 \%$ de pertinência prática; e 0,98 para linguagem (semântica e apropriação para a idade dos participantes), valores todos considerados excelentes (Landis \& Koch, 1977). Os coeficientes de validade de conteúdo obtiveram-se os seguintes resultados: 0,94 para linguagem; 0,94 para a pertinência prática; e 0,99 para a pertinência teórica. Com esses resultados, considera-se que esta adaptação do RESTQ-Sport possui evidências de validade de conteúdo suficientes para ser utilizada com bailarinos adolescentes.

\section{Evidências de validade baseadas na estrutura interna do RESTQ-Dance}

Com base na análise paralela para variáveis policóricas, foram obtidos até quatro fatores com eigenvalue expressivos não evidenciados ao acaso. Em seguida, procedeu-se à análise fatorial exploratória, com índices confirmatórios, via software MPLUS, forçando-se soluções de um a quatro fatores, utilizando a rotação oblíqua geomin, e método de extração Maximum Likelihood Robust (MLR), considerado como um método robusto adequado para variáveis policóricas. Das soluções investigadas (de 1 a 4 fatores), a solução de três fatores foi a que apresentou maior coerência teórica para o agrupamento dos itens. Os índices de ajuste dessa solução foram $\mathrm{X}^{2} / \mathrm{df}=1,89$ (bom); RMSEA $=0,071$ (aceitável); $\mathrm{CFI}=0,515$ (insatisfatório); $\mathrm{e}$, SMR $=0,074$ (bom), de acordo com pontos de corte de Hooper, Coughlan e Mullen (2008). A partir dos dados apresentados, foi mantida a estrutura de quatro fatores. As cargas fatoriais encontradas, o número de itens mantidos por fator e os índices de consistência interna (alfa de Cronbach) estão na Tabela 1 (os itens que permaneceram em cada um dos fatores estão em negrito).

Tabela 1. Fatores Encontrados com Respectivas Cargas e Índices de Consistência Interna do RESTQ-Dance.

\begin{tabular}{|c|c|c|c|}
\hline Itens & Estresse geral & Recuperação & Estresse específico \\
\hline 3 & 0,13 & $\underline{0,44}$ & $-0,06$ \\
\hline 4 & $\underline{0.44}$ & 0,03 & $-0,04$ \\
\hline 5 & $\underline{0,52}$ & $-0,16$ & 0,02 \\
\hline 6 & $-0,12$ & $\underline{0.45}$ & $-0,03$ \\
\hline 7 & $\underline{0,560}$ & 0,01 & $-0,18$ \\
\hline 8 & $\underline{0.62}$ & $-0,09$ & $-0,04$ \\
\hline 10 & $-0,17$ & $\underline{0.50}$ & 0,09 \\
\hline 11 & $\underline{0.51}$ & $-0,03$ & $-0,08$ \\
\hline 12 & $\underline{0,45}$ & 0,11 & $-0,04$ \\
\hline 14 & $-0,12$ & $\underline{0.53}$ & $-0,03$ \\
\hline 15 & $\underline{0.52}$ & 0,03 & $-0,07$ \\
\hline 16 & $\underline{0.53}$ & $-0,01$ & 0,12 \\
\hline 17 & $-0,01$ & $\underline{0.49}$ & $-0,09$ \\
\hline 18 & $\underline{0,39}$ & 0,16 & 0,26 \\
\hline 19 & $-0,14$ & $\underline{0.41}$ & $-0,18$ \\
\hline 20 & $\underline{0,60}$ & 0,08 & 0,02 \\
\hline 21 & $\underline{0.62}$ & $-0,01$ & 0,02 \\
\hline 22 & $\underline{0,50}$ & $-0,21$ & 0,23 \\
\hline 23 & 0,06 & $\underline{0.48}$ & $-0,16$ \\
\hline 24 & $\underline{0,61}$ & $-0,11$ & 0,04 \\
\hline 25 & $\underline{0.56}$ & 0,22 & $-0,02$ \\
\hline 26 & $\underline{0,51}$ & 0,07 & 0,24 \\
\hline 29 & 0,06 & $\underline{0.49}$ & $-0,17$ \\
\hline
\end{tabular}




\begin{tabular}{|c|c|c|c|}
\hline 30 & $\underline{0.63}$ & 0,03 & $-0,00$ \\
\hline 31 & $\underline{0.43}$ & $-0,25$ & 0,25 \\
\hline 33 & $-0,09$ & $\underline{0.55}$ & 0,09 \\
\hline 34 & 0,01 & $\underline{0,65}$ & $-0,23$ \\
\hline 35 & $\underline{0.40}$ & 0,26 & $-0,06$ \\
\hline 36 & $\underline{0,53}$ & 0,14 & $-0,01$ \\
\hline 37 & $\underline{0.46}$ & $-0,09$ & 0,39 \\
\hline 38 & 0,13 & $\underline{0,47}$ & $-0,25$ \\
\hline 39 & 0.67 & $-0,21$ & 0,04 \\
\hline 40 & $\underline{0,55}$ & $-0,17$ & 0,23 \\
\hline 41 & $\underline{0.39}$ & 0,06 & $-0,05$ \\
\hline 42 & 0,37 & $-0,01$ & $\underline{0.45}$ \\
\hline 43 & $-0,01$ & 0.62 & $-0,37$ \\
\hline 44 & $\underline{0,40}$ & 0,16 & $-0,16$ \\
\hline 45 & $\underline{0.51}$ & $-0,24$ & 0,20 \\
\hline 46 & 0,31 & $-0,05$ & $\underline{0.55}$ \\
\hline 47 & 0,07 & $\underline{0.60}$ & $-0,26$ \\
\hline 48 & 0,35 & $-0,02$ & $\underline{0.41}$ \\
\hline 51 & 0,28 & $-0,01$ & $\underline{0.49}$ \\
\hline 52 & $-0,07$ & $\underline{0.45}$ & 0,18 \\
\hline 53 & 0,10 & $\underline{0,35}$ & 0,03 \\
\hline 54 & 0,31 & $-0,06$ & $\underline{0.55}$ \\
\hline 55 & $-0,03$ & $\underline{0,48}$ & 0,29 \\
\hline 56 & 0,08 & $\underline{0.40}$ & 0,00 \\
\hline 58 & 0,24 & $-0,06$ & $\underline{0,45}$ \\
\hline 59 & 0,05 & 0,23 & $\underline{0.41}$ \\
\hline 60 & $-0,03$ & $\underline{0,36}$ & 0,30 \\
\hline 61 & $-0,03$ & $\underline{0.31}$ & 0,26 \\
\hline 62 & 0,04 & $\underline{0,58}$ & $-0,26$ \\
\hline 65 & 0,15 & $\underline{0.56}$ & $-0,16$ \\
\hline 66 & $\underline{0,35}$ & 0,10 & 0,25 \\
\hline 67 & 0,20 & $\underline{0.34}$ & $-0,09$ \\
\hline 68 & 0,34 & $-0,20$ & $\underline{0.50}$ \\
\hline 69 & $-0,06$ & $\underline{0.38}$ & 0,08 \\
\hline 70 & 0,03 & 0,57 & 0,15 \\
\hline 71 & 0,15 & $\underline{0.46}$ & $-0,20$ \\
\hline 73 & 0,31 & $-0,05$ & $\underline{0.51}$ \\
\hline 74 & $-0,01$ & $\underline{0.41}$ & 0,11 \\
\hline 75 & $-0,01$ & $\underline{0,31}$ & $-0,14$ \\
\hline 76 & 0,18 & $-0,28$ & $\underline{0.45}$ \\
\hline № de tens & 26 & 27 & 10 \\
\hline a & 0,92 & 0,91 & 0,79 \\
\hline
\end{tabular}

Nota. Em negrito e sublinhado está a carga do item no fator em que ele foi considerado.

Pode-se observar na Tabela 1 que foi selecionado um total de 63 itens para o instrumento, que passou para a denominação de Recovery-Stress Questionnaire for Dancers - RESTQ-Dance. Para esta escolha do nome do instrumento, baseou-se na validação que o instrumento feita para análise de estresse e recuperação de técnicos, o qual foi denominado RESTQ-Coach (V. J. Costa, Gomes, Andrade, \& Samulski, 2012).

Foram utilizados os seguintes critérios para exclusão dos itens em cada fator: (a) o item prejudicar ou não favorecer a consistência interna do fator; (b) pouca consistência interpretativa para manutenção do item no fator; (c) cargas expressivas em outros fatores (diferença menor que 0,50 nas cargas intrafatores). Com base nesses critérios, chegou-se ao conjunto final de itens para o instrumento. Em relação à consistência interna dos fatores encontrados, verifica-se uma variação entre 0,79 e 0,92 para os fatores, sendo que para o conjunto total foi de 0,91. Uma vez que a estrutura interna do instrumento foi definida, na continuidade, poderão ser calculados os valores correspondentes ao Estresse Geral - voltado para o contexto geral, social, e amplificado da vida do atleta; Estresse Específico - caracterizado por estressores relacionados à prática da dança e de competições; e por fim, Recuperação - relacionado aos mecanismos de adaptação, descanso e recuperação aos estressores que os bailarinos utilizaram nos últimos três dias.

O Fator 1 (26 itens; $a=0,92$ ) foi denominado de Estresse Geral por apresentar itens que representam reações de estresse mais amplas, sociais, não necessariamente voltadas ao contexto de atividade física. São exemplos de itens desse fator: item 5 - Qualquer coisa me incomodava; item 22 - Eu estava aborrecido(a) com outras pessoas. O Fator 2 (27 itens; $a=0,91$ ), denominou-se Recuperação, pois apresenta situações de enfrentamento ao estresse ou mesmo percepção sobre a própria recuperação física e psíquica. São exemplos de itens desse fator: item 23 - Eu me encontrei com alguns amigos; item 53 - Eu me recuperei bem fisicamente. Por fim, o Fator 3 (10 itens; $a=0,79$ ) denominou-se Estresse Específico por apresentar reações específicas da atividade física praticada, dança. São exemplos de itens desse fator: item 54 - Eu me senti esgotado(a) na dança; item 68 - Eu quis abandonar a dança; item 73 - Eu senti que estava próximo de me machucar.

\section{Discussão}

Há uma ampla gama de instrumentos de avaliação voltados à área da Psicologia do Esporte, mas é restrito o número de adaptações validadas especificamente para diferentes modalidades esportivas (Peixoto \& Nakano, 2014; Rubio, 2011; Silva et al., 2014). Portanto, existe uma lacuna de conhecimento na investigação de instrumentos específicos aplicados na área atualmente, além da pouca atenção para sua adequação às diferentes áreas do esporte. Nesse sentido, a Psicometria da atividade física pode contribuir nesse campo (Rubio, 2007). Da mesma forma e com maior carência de instrumentos, afirma-se a importância dessa 
discussão no contexto pré-competitivo de bailarinos (Silva, 2016; Silva \& Enumo, 2016; Silva et al., 2013), os quais têm altos níveis de exigências físicas e mentais, gerando altos níveis de estresse e/ou overtraining e overreaching (Constantino et al., 2010).

Desse modo, a adaptação do RESTQ-Sport para o contexto da dança competitiva se justifica, tanto na falta de um instrumento voltado para a avaliação dos fatores de estresse e recuperação para essa população em específico no Brasil, como na própria relevância de tal instrumento para a avaliação. No processo de adaptação, buscou-se adequar o vocabulário à população de modo a não comprometer a compreensão, tanto no que diz respeito ao caráter da dança, quanto à faixa etária da adolescência. Além disso, foram utilizados os critérios propostos por Primi et al. (2009) de novas definições de validade denominadas como "evidências de validade", verificando o conteúdo frente aos juízes e a consistência interna frente a análises fatoriais. Não foram, porém, buscadas as evidências de validade baseadas no processo de resposta, nas relações com variáveis externas e em consequências de testagem, questões essas que podem vir a ser contempladas em futuros estudos.

Em relação às evidências de validade de consistência interna, houve grandes alterações do instrumento original, voltado para atletas, para esta adaptação para bailarinos. Primeiramente, houve diminuição de 14 itens do instrumento, passando de 77 itens (no original) para 63 (versão adaptada para bailarinos). Isso ocorreu para viabilizar a interpretação dos resultados agrupados, bem como para melhorar o nível de precisão do instrumento. A principal diferença ocorreu no número de fatores existentes no instrumento, diminuindo de 19 para três. Esse resultado é relevante, pois indica que esta versão do instrumento para bailarinos não contém muitas divisões conceituais, de forma que o relevante nesta população é a diferenciação entre estresse geral, estresse específico e recuperação. Esta configuração é coerente com o objetivo do instrumento, que é medir os níveis de estresse e recuperação de seus participantes analisados (L. O. Costa \& Samulski, 2005).

Esta diferença de organização do instrumento reforça a ideia argumentada por McCann et al. (2011) de que os instrumentos psicológicos precisam ser construídos e ter os resultados analisados com base na especificidade da modalidade de atividade física/ esportiva praticada. Essas recomendações decorrem das diferenças particulares de treinamento na dança, as quais podem levar a alterações psicológicas diferenciadas, e também dos aspectos cognitivos, uma vez que se está lidando com adolescentes e com sua compreensão dos itens pelos participantes, já que os mesmos eram adolescentes. Entretanto, o fator faixa etária foi previamente analisado e adequado no processo de evidências de validade de conteúdo, contando-se com a colaboração de juízes especialistas, como proposto por Balbinotti et al. (2007), Gallahue e Ozmun (2003) e Pasquali (2004).

As diferenças entre a versão para atletas e para bailarinos poderiam decorrer do tamanho desta amostra $(N=264)$; mas, este estudo contou quase o dobro de participantes do próprio estudo de validação original do instrumento para população brasileira ( $N=134$ atletas de diferentes modalidades esportivas). Além disso, alguns autores consideram que acima de 200 participantes já é possível fazer a verificação adequada das dimensões do instrumento e do seu nível de precisão (Nunnally, 1978).

Deve-se ressaltar que todos os cuidados metodológicos foram assegurados, como avaliação de evidências de validade por juízes especialistas, treinamento para os aplicadores do instrumento em período de coleta de dados, bem como checagem do índice Kappa, para verificar a consistência intraavaliadores, usando-se métodos estatísticos recentes e robustos, condições estas que garantem maior precisão dos resultados. Por fim, os valores de consistência interna, com o alfa de Cronbach variando de 0,79 a 0,92, demonstram que o instrumento apresenta estrutura adequada e fidedigna, já que o valor de precisão de corte é 0,70 (Dancey \& Reidy, 2008). Assim, os itens adaptados para o RESTQ-Dance se mostraram adequados e internamente consistentes, conforme mostram os resultados obtidos pela análise fatorial e os coeficientes de alfa de Cronbach, além de passar pela aprovação de juízes referentes à validação de conteúdo, algumas considerações devem ser feitas. É importante notar a presença de fatores com poucos itens, estando desequilibrados perante outros fatores com mais itens. Contudo, o cálculo final proposto pela literatura, através de médias, minimizaria esse problema. Assim, ao observarem-se os altos índices de validade e fidedignidade obtidos nos resultados, é possível notar que o instrumento é passível de uso, já que todos os valores foram acima de 0,70.

No estudo de tradução e validação do RESTQSport para a língua portuguesa, L. O. Costa e Samulski (2005) encontraram um índice adequado de consistência interna do instrumento e confiabilidade substancial em 
16 das 19 escalas ( $a \geq 0,70)$. O presente estudo, embora com modificações dos fatores, também encontrou índices de confiabilidade, o que é fundamental para considerar que determinado teste mede o construto que se pretende mensurar. Considerando os resultados de ambos os estudos de validação - o de L. O. Costa e Samulski (2005) com diferentes modalidades esportivas e este, que pretendeu validar o RESTQ-Sport para a amostra de dançarinos adolescente -, entende-se que o instrumento é confiável para mensurar o estresse e a recuperação.

Embasando-se na estrutura final do instrumento, com 63 itens divididos em três fatores, refletiu-se sobre a forma de cálculo ideal para se conhecer o resultado de estresse e recuperação dos bailarinos. Kellmann, Kalus, Samulski, Costa e Símola (2009) sugerem que os valores das escalas sejam calculados pelos valores médios dos respectivos itens. E, apesar de, neste caso, existirem fatores diferentes, a forma de ser calculado parece ser coerente também aqui. Além disso, essa forma de cálculo minimizaria o efeito negativo do desequilíbrio de itens entre os fatores (já que o Fator 3 apresentou apenas 10 itens, ao contrário dos outros dois fatores que possuíram 26 e 27 itens cada). Kellman et al. (2009) explicam que, em geral, baixos escores em áreas relacionadas com estresse e altos escores relacionados com recuperação são considerados positivos. Os mesmos autores consideram que devido à variação da escala ir de 0 a 6, valores acima de 4 é considerado alto, abaixo de 2 é considerado baixo e entre 2,01 e 3,99 é considerado moderado, seja para estresse específico, estresse geral ou recuperação. Assim, com o conhecimento do perfil do bailarino em relação ao estresse e recuperação, é possível traçar intervenções coerentes com as individualidades presentes em um grupo de dança.

Considera-se como limitação do estudo o número de participantes. Pode-se considerar que a extensão do instrumento tenha sido responsável pela exclusão de muitos participantes do estudo (de 410 para 264), já que muitos deixaram itens em branco, sem responder. Em termos psicométricos, para o cálculo estatístico, o tamanho da amostra é suficiente para se ter uma robustez de resultados; entretanto, é necessário levar em consideração que instrumentos longos podem implicar em perda amostral.

Concluindo, os resultados da validação da escala Recovery-Stress Questionnaire for Dancers (RESTQ-Dance) evidenciam diferenças resultantes das especificidades de uma população de atletas e bailarinos. Torna-se, portanto, imprescindível salientar a importância da validação de instrumentos psicológicos de acordo com a modalidade esportiva realizada ou mesmo atividades físicas específicas, como é o caso da dança, visando a diminuir a "generalização" ou adaptação sem validação dos instrumentos hoje utilizados.

Novas pesquisas sobre evidências de validade podem ser necessárias, por ser este um processo contínuo. Contudo, nesta etapa, o RESTQ-Dance se apresenta como um instrumento que pode ajudar na identificação de indicadores de estresse em dançarinos jovens, avaliação esta que pode colaborar na prevenção dos efeitos negativos do estresse sobre a saúde desses adolescentes.

\section{Referências}

Alves, R. N., Costa, L. O. P., \& Samulski, D. M. (2006). Monitoramento e prevenção do supertreinamento em atletas. Revista Brasileira de Medicina do Esporte, 12(5), 291-296. doi: 10.1590/S151786922006000500013

American Educational Research Association, American Psychological Association, \& National Council for Measurement in Education. (2014). Standars for educational and psychological testing. Washington DC: American Psychological Association.

Associação Brasileira de Empresas de Pesquisa (2013). Critério de classificação econômica Brasil. Recuperado de http://www.abep. org/criterio-brasil

Balbinotti, M. A., Benetti, C., \& Terra, P. R. S. (2007). Translation and validation of the Graham-Harvey survey for the Brazilian context. International Journal of Managerial Finance, 3(1), 26-48. doi: 10.1108/17439130710721644

Barros, J. C., \& De Rose Junior, D. (2006). Situações de stress na natação infanto-juvenil: atitudes de técnicos e pais, ambiente competitivo e momentos que antecedem a competição. Revista Brasileira de Ciência e Movimento, 14(4), 79-86. Recuperado de: http://portalrevistas. ucb.br/index.php/RBCM/article/viewFile/719/723.

Breinbauer, C., \& Maddaleno, M. (2008). Nova abordagem para classificar os estágios de desenvolvimento do adolescente. In C. Breinbauer \& M. Madaleno (Orgs.), Jovens: escolhas de mudanças: promovendo comportamentos saudáveis (pp. 212-221). São Paulo: Roca.

Brink, M. S., Visscher, C., Arends, S., Zwerver, J., Post, W. J., \& Lemmink, K. A. (2010). Monitoring stress and recovery: new insights for the prevention of injuries and illnesses in elite youth soccer players. British Journal of Sports Medicine, 44(11), 809-815. doi: 10.1136/ bjsm.2009.069476

Constantino, A., Prado, W., \& Lofrano-Prado, M. (2010). Ansiedade em bailarinos profissionais nas apresentações de dança. Conexões: Revista da Faculdade de Educação Física da UNICAMP, 8(3), 146-155. Recuperado de: http://fefnet178.fef.unicamp.br/ojs/index. php/fef/article/view/549

Costa, L. O., \& Samulski, D. M. (2005). Processo de validação do Questionário de Estresse e Recuperação para Atletas (RESTQ-Sport) na Língua Portuguesa. Revista Brasileira de Ciência \& Movimento, 13(1), 79-86. Recuperado de https://portalrevistas.ucb.br/index.php/ RBCM/article/download/615/627 
Costa, V. J., Gomes, C. M. A., Andrade, A. G. P., \& Samulski, D. M. (2012).Validação das propriedades psicométricas do RESTQ-Coach na versão brasileira. Motriz, 18(2), 218-232. doi: 10.1590/S198065742012000200002

Cunha, G. S., Ribeiro, J. L., \& Oliveira, A. R. (2006). Sobretreinamento: teorias, diagnóstico e marcadores. Revista Brasileira de Medicina do Esporte, 12(5), 297-302. doi: 10.1590/S1517-86922006000500014

Cunha, R. A., Morales, J. C. P., \& Samulski, D. M (2008). Níveis de estresse e recuperação nos atletas da equipe brasileira de pentatlo aeronáutico militar: um estudo piloto. Revista Mineira de Educação Física, 16(1), 5-22. Recuperado de http://www.revistamineiraefi.ufv. br/artigos/artigos. php?acao=ler\&id=66

Dancey, C. P., \& Reidy, J. (2008). Estatística sem matemática para Psicologia ( $3^{\underline{a}}$ ed., E. Viali, Trad.). Artmed: São Paulo.

Fletcher, D., Hanton, S., Mellalieu, S. D., \& Neil, R. (2012). A conceptual framework of organizational stressors in sport performers. Scandinavian Journal of Medicine \& Science in Sports, 22(4), 545-557. doi: 10.1111/j.1600-0838.2010.01242.x.

Freitas, V. H., Souza, E. A., Oliveira, R. S., Pereira, L. A., \& Nakamura, F.Y. (2014). Efeito de quatro dias consecutivos de jogos sobre a potência muscular, estresse e recuperação percebida, em jogadores de futsal. Revista Brasileira de Educação Física e Esporte, 28(1), 23-30. doi: 10.1590/S1807-55092014005000002

González-Boto, R., Salguero, A., Tuero, C., \& Márquez, S. (2009). Validez concurrente de la versión española del Cuestionario de Recuperación-Estrés para Deportistas (RESTQ-Sport). Revista de Psicología del Deporte, 18(1), 53-72. Recuperado de: http://ddd. uab.cat/record/62842.

Guarino, L. (2015). Is dance a sport? A twenty-first-century debate. Journal of Dance Education, 15(2), 77-80. doi: 10.1080/15290824.2015.978334

Hayton, J. C., Allen, D. G., \& Searpello, V. (2004). Factor retention decisions in exploratory factor analysis: a tutorial on parallel analysis. Organizational Research Methods, 7(2), 191-205. doi: 10.1177/1094428104263675.

Hooper, D., Coughlam, J., \& Muller, M. (2008). Structural equation modeling: guidelines for determining model fit. Business Research Methods, 6(1), 53-60. Recuperado de http://www.ejbrm.com/ volume6/issue 1

Jürimäe, J., Mäestu, J., Purge, P., \& Jürimäe, T. (2004). Changes in stress and recovery after heavy training in rowers. Journal of Science and Medicine in Sport, 7(3), 335-339. doi: 10.1016/S14402440(04)80028-8

Kellmann, M., Altenburg, D., Lormes, W., \& Steinacker, J. M. (2001). Assessing stress and recovery during preparation for the world championships in rowing. Sport Psychologist, 15(2), 151-167. doi: 10.1123/tsp.15.2.151

Kellmann, M., \& Günther, K. D. (2000). Changes in stress and recovery in elite rowers during preparation for the Olympic Games. Medicine and Science in Sports and Exercise, 32(3), 676-683. Recuperado de http://journals.Iww.com/acsm-msse/Fulltext/2000/03000/Changes_in_stress_and_recovery_in_elite_rowers.19.aspx

Kellmann, M., \& Kallus, K. W. (2001). Recovery-stress questionnaire for athletes: use manual. Champaign, Illinois: Human Kinetics

Kellmann, M., Kallus, W. K, Samulski, D. M, Costa, L. O. P, \& Simolla, R. A. P. (2009). Questionário de stress e recuperação para atletas (RESTQ-76 Sport): manual do usuário. Belo Horizonte: Escola de Educação Física, Fisioterapia e Terapia Ocupacional.
Koutedakis, Y., Myszkewycz, L., Soulas, D., Papapostolou, V., Sullivan, I., \& Sharp, N. C. (1999). The effects of rest and subsequent training on selected physiological parameters in professional female classical dancers. International Journal of Sports Medicine, 20(6), 379-383. doi: 10.1055/s-2007-971148

Landis, J. R., \& Koch, G. G. (1977). The measurement of observer agreement for categorical data. Biometrics, 33(1), 159-174. doi: $10.2307 / 2529310$

Lazarus, R. S., \& Folkman, S. (1984). Stress, appraisal and coping. Nova lorque: Springer.

Machado, S. C., Matos, F. O., \& Samulski, D. M. (2011). Análise dos estados de estresse e recuperação de participantes de grupos de corrida: um estudo-piloto. Lecturas: Educación Física y Deportes, 16(156), 1-5. Recuperado de http://www.efdeportes.com/efd156/ estresse-de-participantes-de-grupos-de-corrida.htm

Matos, F. O., Samulski, D. M., Lima, J. R. P. de, \& Prado, L. S. (2014) Cargas elevadas de treinamento alteram funções cognitivas em jogadores de futebol. Revista Brasileira de Medicina do Esporte, 20(5), 388-392. doi: 10.1590/1517-86922014200501274

McCann, S. C., Jowdy, D. P., \& Van Raalte, J. L. (2011). Avaliação em Psicologia do Esporte. In J. L. Van Raalte \& B. W. Brewer (Orgs.), Psicologia do esporte (2 ${ }^{\underline{a}}$ ed., pp. 291-305, H. T. Buchup, Trad.). São Paulo: Santos Editora.

Meehan, H. L., Bull, S. J., Wood, D. M., \& James, D. V. (2004). The overtraining syndrome: a multicontextual assessment. The Sports Psychologist, 18(2), 154-171. doi: 10.1123/tsp.18.2.154

Nogueira, F. C. A., Nogueira, R. A., Miloski, B., Cordeiro, A. H. O., Werneck, F. Z., \& Bara Filho, M. (2015). Influência das cargas de treinamento sobre o rendimento e os níveis de recuperação em nadadores. Revista da Educação Física/UEM, 26(2), 267-278. doi:10.4025/reveducfis.v26i2.23120

Nunnally, J. C. (1978). Psychometric theory. Nova lorque: McGraw-Hill.

Pasquali, L. (2004). Psicometria: teoria dos testes na Psicologia e na Educação. Petrópolis: Vozes.

Peixoto, E. M., \& Nakano, T. C. (2014). Problemas e perspectivas na utilização dos testes psicológicos em Psicologia do Esporte. In C. R. Campos \& T. C. Nakano (Org.), Avaliação psicológica: direcionada a populações específicas (pp. 201-232). São Paulo: Vetor Ed.

Primi, R., Muniz, M., \& Nunes, C. H. (2009). Definições contemporâneas de validade. In C. S. Hutz (Org.), Avanços e polêmicas em avaliação (pp. 243-265). São Paulo: Casa do Psicólogo.

Rohleder, N., Beulen, S. E., Chen, E., Wolf, J. M., \& Kirschbaum, C. (2007). Stress on the dance floor: the cortisol stress response to social-evaluative threat in competitive ballromm dancers. Personality and Social Psychology Bulletin, 33(1), 69-84. doi: $10.1177 / 0146167206293986$

Rubio, K. (2007). Da Psicologia do Esporte que temos à Psicologia do Esporte que queremos. Revista Brasileira de Psicologia do Esporte, 1(1), 1-13. Recuperado de de http://pepsic.bvsalud.org/scielo.php?script=sci_ arttext\&pid=S1981-91452007000100007\&lng=pt\&tlng=pt.

Rubio, K. (2011). A avaliação em Psicologia do Esporte e a busca de indicadores de rendimento. In L. F. Angelo \& K. Rubio (Orgs.), Instrumentos de avaliação em Psicologia do Esporte (pp. 13-26). São Paulo: Casa do Psicólogo.

Shonkoff, J. P., Garner, A. S., Siegel, B. S., Dobbins, M. I., Earls, M. F., McGuinn, L., ... Wood, D. L. (2012). The lifelong effects of early childhood adversity and toxic stress. Pediatrics, 129(1), e232-e246. doi: 10.1542/peds.2011-2663 
Silva, A. M.B. (2016). Uma proposta de avaliação e intervenção psicológica no enfrentamento da dor em bailarinos (Tese de Doutorado não publicada). Pontifícia Universidade Católica de Campinas, Campinas, SP.

Silva, A. M. B., \& Enumo, S. R. F. (2016). Avaliação psicológica na dança: instrumentos e possibilidades. In E. M. Peixoto, T. C. N. Primi, \& M. A. A. Balbinotti (Orgs.), Novas perspectivas em avaliação psicológica na Psicologia do Esporte e do Exercício Físico (pp. 185-218). Curitiba: CRV.

Silva, A. M. B., Guimarães, C. A., Foch, G. F. L., \& Enumo, S. R. F. (2014). Instrumentos aplicados em estudos brasileiros em Psicologia do Esporte. Estudos Interdisciplinares em Psicologia, 5(2), 77-95. doi: 10.5433/2236-6407.2014v5n2p77

Silva, A. M. B., Tkac, C. M., \& Enumo, S. R. F. (2013). Ansiedade pré-competição e concentração em bailarinos de jazz. Lecturas: Educación Física y Deportes. 18(183). Recuperado de http://www.efdeportes. com/efd183/ansiedade-e-concentracao-em-bailarinos-de-jazz.htm

Sousa, F. N., Mariani, M. E., \& Samulski, D. M. (2004). Análise do nível de estresse e da ansiedade em bailarinos e bailarinas profissionais na pré-estréia de um espetáculo de dança. Revista On-Line Unileste, 1(1), 1-6.
Tricoli, V. A. C. (2014). Diagnóstico do stress na adolescência. In M. E. N. Lipp (Org.), Stress em crianças e adolescentes (pp. 59-76). Campinas, SP: Papirus.

Urhausen, A., \& Kindermann, W. (2002). Diagnosis of overtraining. Sports Medicine, 32(2), 95-102. doi: 10.2165/00007256200232020-00002.

Uusitalo, A. L. (2001). Overtraining. Making a difficult diagnosis and implementing targeted treatment. The Physician and Sportmedicine, 29(5), 35- 50. doi: 10.3810/psm.2001.05.774.

Verardi, C. E. L., Miyazaki, M. C. O. S., Nagamine, K. K., Lobo, A. P. S., \& Domingos, N. A. M. (2012). Esporte, stress e burnout. Estudos de Psicologia, 29(3), 305-313. doi: 10.1590/S0103$-166 \times 2012000300001$

Verderi, É. B. (1998). Dança na escola. Rio de Janeiro: Sprint.

Watkins, M. W. (2006). Determining parallel analysis criteria. Journal of Modern Applied Statistical Methods, 5(2), 344-346. Recuperado de http://digitalcommons.wayne.edu/jmasm/vol5/iss2/8/ 
Andressa Melina Becker da Silva, Doutora em Psicologia pela Pontifícia Universidade Católica de Campinas (PUC-Campinas), é Professora Adjunta da Universidade de Sorocaba (UNISO) na Graduação de Psicologia. Endereço para correspondência: Rodovia Raposo Tavares - Vila Artura, Sorocaba - SP. CEP 18023-000. Telefone:

(19) 98400-7922. E-mail andressa.silva@prof.uniso.br.

Sônia Regina Fiorim Enumo, Doutorado em Psicologia Experimental pela Universidade de São Paulo (USP), Pós-doutorado na Universidade

Estadual de Campinas (UNICAMP), é Professora A2 da Pontifícia Universidade Católica de Campinas (PUC-Campinas), no Programa de Pós-Graduação em Psicologia e no Programa de Pós-Graduação em Ciências da Saúde. E-mail: sonia.enumo@puc-campinas.edu.br

Murilo Fernandes de Araújo, Graduando em Psicologia pela Pontifícia Universidade Católica de Campinas (PUC-

Campinas). E-mail: murilo.fa@puc-campinas.edu.br

Lucas de Francisco Carvalho, Doutor em Psicologia com ênfase em Avaliação Psicológica na Universidade São Francisco (USF), é Professor na Universidade São Francisco no Programa de PósGraduação em Psicologia. E-mail: lucas@labape.com.br

Isabella Goulart Bittencourt, é Psicóloga graduada pela Universidade Federal Santa Catarina (UFSC). E-mail: isabellagoulartb@gmail.com

Renan de Morais Afonso, Graduando em Psicologia pela Pontifícia Universidade Católica de Campinas (PUCCampinas). E-mail: renan.ma@puccampinas.edu.br

Tatiane Stephan Rocchetti Luz, Concluinte na Graduação em Psicologia pela Pontifícia Universidade Católica de Campinas

(PUC-Campinas). E-mail: tatiane.srl@puccampinas.edu.br 